\title{
A Powerful Transformation Rule, its Applications and Variants
}

\author{
Klaus Achatz and Helmuth Partsch \\ Department of Computer Science, University of Ulm, \\ 89069 Ulm, Germany \\ E-mail: \{achatz,partsch\}@informatik.uni-ulm.de, \\ Tel: +49 731 502-4161, Fax: -4162
}

\begin{abstract}
This paper introduces, discusses and proves a transformation rule to convert specifications of set-valued functions defined by set comprehension into functional implementations. The power of the rule is illustrated by several examples, among them a Prolog interpreter. Also, variants of the rule for specifications involving existential quantification and arbitrary choice are presented and illustrated by representative examples.
\end{abstract}

\section{Keywords}

Transformational programming, backtracking, set comprehension, functional implemention

\section{INTRODUCTION}

Transformational programming is short for a methodology of constructing programs from formal problem specifications by stepwise application of formal, semantics-preserving transformation rules. In the following the reader is assumed to be basically familiar with the idea of transformational programming and its basic principles. A brief introduction and an overview can be found, e.g., in (Bauer et al., 1989) or (Boiten et al., 1992). For a comprehensive treatment of the subject, cf. (Partsch, 1990).

In this paper we mainly introduce a powerful transformation rule to convert specifications of set-valued functions defined by set comprehension into executable functional implementations.

The general form of such a specification reads

$$
\begin{aligned}
& f(X) \text { where } \\
& f: \mathbf{m} \rightarrow \text { set of } \mathbf{n} \\
& f(x)=\text { def }\{y: \mathbf{n} \mid \mathrm{Q}(x, y)\} .
\end{aligned}
$$


It asks for computing the value of a function $f$ for some initial argument value $X$ of type $\mathbf{m}$, where $f$ computes the set of all $y$ of type $\mathbf{n}$ such that the argument of $f$ and $y$ are related by some predicate $Q$.

\section{Example:}

A typical instance of such a specification is provided by the $\mathrm{N}$-Queens problem which asks for finding all ways to place $N$ mutually non-attacking queens on an $N \times N$ chessboard:

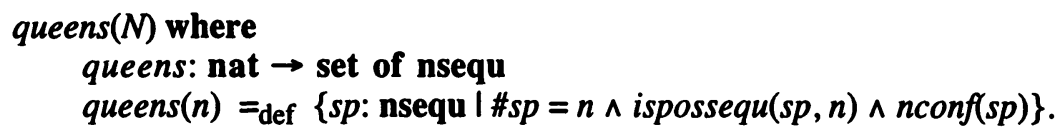

Here

$$
\text { nsequ }=_{\text {def }} \text { sequ of nat }
$$

is the type of sequences of natural numbers used to represent positions of queens in the different columns of the chessboard;

$$
\# s p=n
$$

checks whether the length of sequence $s p$ is equal to $n$;

ispossequ: nsequ $\times$ nat $\rightarrow$ bool

ispossequ $(s, n)=$ def $\forall i$ : nat $\mid 1 \leq i \leq \# s \Delta s[i] \leq n$

restricts the elements of nsequ to those less or equal to $n$, and

$$
\begin{aligned}
& \text { nconf: nsequ } \rightarrow \text { bool } \\
& \begin{aligned}
\text { nconf }(s)= & =\operatorname{def} \forall(i, j: \text { nat } \mid 1 \leq i, j \leq \# s \wedge i \neq j) \mid \\
& s[i] \neq s[j] \wedge \operatorname{abs}(s[i]-s[j]) \neq \operatorname{abs}(i-j)
\end{aligned}
\end{aligned}
$$

guarantees that all positions in the different columns are "nonattacking", i.e., do not mutually occupy the same row or the same diagonal.

The rule we will present deals with the above kind of specifications and can be profitably used for all kinds of problems that ask for a set of complex objects that may somehow be built up incrementally, possibly involving backtracking. The rule emerged when dealing with a formal derivation of a Prolog interpreter from a descriptive specification. It turned out that most transformation steps in this concrete exercise were independent of the particular problem and that the remaining ones could be abstracted appropriately. Meanwhile, the rule has been successfully applied to numerous non-trivial examples, some of which are presented in this 
paper. More examples and a detailed treatment of those presented here can be found in (Achatz, Partsch, 1996).

\subsection{Notational conventions}

Within our rules and examples, we use the following notational conventions.

\subsubsection{Fonts}

Italic is used for all bound variables. Scheme parameters (for expressions in rules) are denoted by upright-uppercase letters (augmented with "arguments" to indicate potential dependencies). And for types (and type variables) we employ boldfacelowercase symbols.

\subsubsection{Data types}

Type variables (in program schemes) are denoted by single boldface letters (such as $\mathbf{m}$ or $\mathbf{n}$ ). As basic types, we assume to have available natural numbers (with type nat and the usual operations) as well as booleans.

Based on basic types, more complex ones may be built by forming supertypes (e.g., $\mathbf{m}$ I $\mathbf{n}$ comprising all elements of types $\mathbf{m}$ and $\mathbf{n}$ ) and record types (e.g., $(a: \mathbf{m}, b: \mathbf{n})$ denoting pairs of elements of types $\mathbf{m}$ and $\mathbf{n}$ respectively, where $a$ and $b$ are selectors for the first (second) argument).

More complex types we will use are sets, sequences, and maps. For respective formal definitions in terms of algebraic types we refer to (Partsch, 1990). The intuition of the basic operations of these types (appearing in the examples) is as follows:

sets (typ set of) with operations

\begin{tabular}{|c|c|}
\hline $\begin{array}{l}\cdot \varnothing \\
\cdot \cup \\
\cdot \quad \in\end{array}$ & $\begin{array}{l}\text { the empty set } \\
\text { set union } \\
\text { subset relation } \\
\text { element relation }\end{array}$ \\
\hline
\end{tabular}

sequences (type sequ of) with operations

- 0 the empty sequence

- hd (lst) first (last) element of a (non-empty) sequence

- tl (fst) (non-empty) sequence without first (last) element

- ++ concatenation of sequences (also used to append single elements)

- $s[i] \quad i$-th element of a (non-empty) sequence $s$

- \#s length of sequence $s$

- $\in$ element relation

maps (correspondences defined by an algebraic type EMAP, cf. [Partsch 90]) with operations
- $\varnothing$
-
the empty map
composition of maps 
- $m[i]$ the "value" of map $m$ for "argument" $i$

- dom the domain of the map

- ran the range of the map

- $m[i] \leftarrow a \quad$ map $m$ with "value" of $i$-th "argument" updated to $a$.

\subsubsection{Programs and program schemes}

For programs and program schemes a mainly self-explanatory Pascal-like notation is used with a strict, call-by-value semantics. For a complete definition of the language used, we refer to (Bauer et al., 1985). Some of the less conventional notations (and their meaning) are:

- $\{a: \mathbf{m} \mid \mathrm{P}(a)\}$

set comprehension, denoting the set of all elements $a$ (of type $\mathrm{m})$ for which $\mathrm{P}(a)$ holds

- some $a: \mathrm{m} \mid \mathrm{P}(a) \quad$ (non-deterministic) choice, denoting some element $a$ (of type $\mathbf{m})$ which satisfies $\mathrm{P}(a)$

- $(\mathbf{m} \mid \mathrm{P}(a))$ subtype of $\mathbf{m}$, comprising all elements $a$ (of type $\mathbf{m}$ ) which fulfil $\mathrm{P}(a)$; also used for assertions in the domain of functions

- $\#\{\ldots\}$

- $\Delta(\nabla)$ cardinality of a set sequential conjunction (disjunction)

As an abbreviation, we omit the type $\mathbf{m}$ where it is obvious.

\subsubsection{Transformation rules}

Transformation rules are denoted as $\overbrace{O}^{I}$ resp.

$I$ is the input scheme of the rule, $O$ its output scheme. The double arrow means semantic equivalence, the single arrow descendance. $C$ is a set of applicability conditions, all of which are (implicitly) universally quantified clauses (consisting of a premise, $t$ as an implicational symbol, and a semantic relation over program schemes - equivalence or descendance - as a consequence). Within applicability conditions, $\boldsymbol{=}$ is used to denote semantic equivalence, and $\subseteq$ to denote descendance. If there is no premise, also + will be left out. The syntactic constraints (following a rule) give sufficient (but not neccessarily minimal) information to infer complete typing of all expressions and functions involved. For details on the logical background of transformation rules, cf. (Partsch, 1990).

To shorten the presentation of the rules we furthermore use the following

\section{General convention}

In all transformation rules definedness and determinacy are assumed for all expression symbols and auxiliary functions. Also, all program schemes are supposed to be syntactically valid and context-correct. 


\subsection{Outline of the following sections}

In Sect. 2 we formally develop our central transformation rule, give some intuition on how to use it, and illustrate the essential steps by means of our running example, the N-Queens problem. Section 3 deals with some applications of the rule. In this context, the treatment of the $\mathrm{N}$-Queens problem will be completed by giving all proof obligations that result from instantiating the applicability conditions of our rule and by sketching how to satisfy them. As another example, the problem of a Prolog interpreter is treated in a condensed form. In Sect. 4 some variants of the rule from Sect. 2 are discussed. These variants deal with other specification constructs (such as existential quantification or non-deterministic choice) and are also illustrated by examples (from the area of parsing). As a particular variant we will also show in Sect. 4 that, under additional constraints, our rule from Sect. 2 indeed simplifies to a form one would intuitively expect. Section 5, finally, deals with related work and draws some conclusions.

\section{A POWERFUL TRANSFORMATION RULE}

In this section, we give a stepwise formal derivation which is based on certain assumptions (called postulate below). At the end, the starting point of this formal derivation, the final result, and all assumptions used in the derivation will be compiled into a corresponding transformation rule.

\subsection{Derivation of the transformation rule}

Our derivation is split up into several phases each of which is given in a calculational style. The single steps of the respective calculations are labelled for later reference. For the details of the basic transformations used in reasoning, we refer the reader to (Partsch, 1990).

The starting point of our derivation is the program scheme

$$
\begin{aligned}
& f(X) \text { where } \\
& f: \mathbf{m} \rightarrow \text { set of } \mathbf{n} \\
& f(x)={ }_{\text {def }}\{y: \mathbf{n} \mid \mathrm{Q}(x, y)\} .
\end{aligned}
$$

Obviously, the definition of queens as given in Sect. 1 matches this scheme and yields the instantiations
$f, X, x, y$
$\mathrm{Q}(x, y)$
by queens, $N, n, s p$
b y $\mathrm{Q}(n, s p) \cong \# s p=n \wedge$ ispossequ $(s p, n) \wedge n \operatorname{conf}(s p)$.

To be able to compute $f$ we require

postulate $(0)(\#\{y: n \mid Q(x, y)\}<\infty)=$ true

which guarantees the finiteness of the set to be computed. 
(a) As a first step, we transform $f(X)$ into $g(X)$ for a suitably defined function $g$. To this end we define

$$
\begin{aligned}
& g: \mathbf{m} \rightarrow \text { set of } \mathbf{n} \\
& g(u)=\operatorname{def} g^{\prime}(u, \mathrm{E}, \emptyset)
\end{aligned}
$$

and

$$
\begin{aligned}
& g^{\prime}:(u: \mathbf{m} \times v: \mathbf{r} \times s: \text { set of } \mathbf{n} \mid \mathrm{P}(X, u, v)) \rightarrow \text { set of } \mathbf{n} \\
& g^{\prime}(u, v, s)=\operatorname{def} s \cup\left\{y: \mathbf{n} \mid Q^{\prime}(u, v, y)\right\} .
\end{aligned}
$$

The intuition behind this step is the idea of "embedding", that is the introduction of a suitably initialized function with additional parameters. The purpose of the additional parameters in $\boldsymbol{g}^{\prime}$ is as follows:

- The parameter $v$ of type $\mathbf{r}$ basically serves to incrementally construct elements of the set aimed at with $\mathrm{E}$ being kind of a neutral element to start with. Type $\mathbf{r}$ is supposed to be a "supertype" of $\mathbf{n}-$ in most applications identical with $\mathbf{n}$ or a product type with a component of type $n$.

- The parameter $s$ of type set of $\mathbf{n}$ is used as an accumulator for the set to be constructed, and, obviously, initialized with $\emptyset$.

$P$ is an invariant (for $g^{\prime}$ ) to couple the additional parameter of type $\mathbf{r}$ to the original one (of type $\mathbf{m}$ ) for which

postulate (1) $\mathrm{P}(X, X, \mathrm{E})=$ true

is required; that is $\mathbf{P}$ holds for the initial values.

Predicate $Q$ ' is a "weaker" form of the original predicate $Q$ that takes the incremental construction of elements of type $r$ into account. In most applications, $Q$ ' will simply be of the form

$$
\mathrm{Q}^{\prime}(u, v, y)=\exists y^{\prime} \mid c\left(v, y^{\prime}\right)=y \Delta \mathrm{Q}\left(u, y^{\prime}\right)
$$

with $c$ being a "constructor" for elements of type $r$.

To establish the relationship between $Q$ and $Q^{\prime}$ we require

postulate $(2) \mathrm{Q}(x, y)=\mathrm{Q}^{\prime}(x, \mathrm{E}, y)$

To establish the equivalence of $f(X)$ with $g(X)$ we calculate as follows:

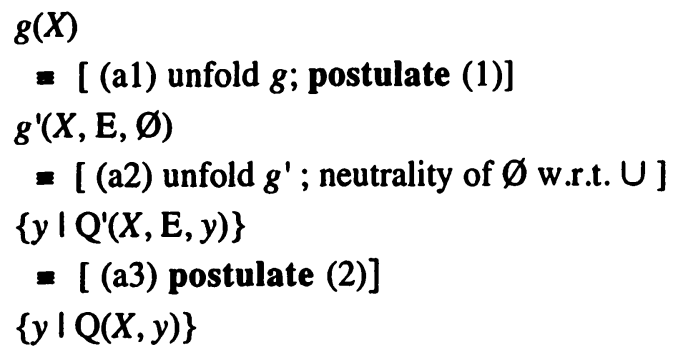


$=[(\mathrm{a} 4)$ fold $f]$

$f(X)$.

Since $f$ has a defined value due to the general convention and postulate $(0)$, so do $g$ and $g^{\prime}$ according to the above reasoning.

Example (cont.):

If we further instantiate

$g, g^{\prime}, u, v \quad$ by $q u, q u^{\prime}, n, p$

and choose to instantiate

$\mathrm{P}(X, u, v) \quad$ by $\mathrm{P}(u, n, p) \cong \# p \leq n \wedge i \operatorname{spossequ}(p, n) \wedge n \operatorname{conf}(p)$

$\mathrm{E} \quad$ by [] (* the empty sequence of columns to start with *)

$\mathrm{Q}^{\prime}(u, v, y) \quad$ by $\mathrm{Q}^{\prime}(n, p, s p) \cong \exists s^{\prime}:$ nsequ $\mid s p=p++s^{\prime} \wedge \mathrm{Q}(n, p)$

(* the existence of a possible extension of $p$ to obtain a

complete sequence of positions with the requested properties *)

we obtain (as an intermediate form) for our running example:

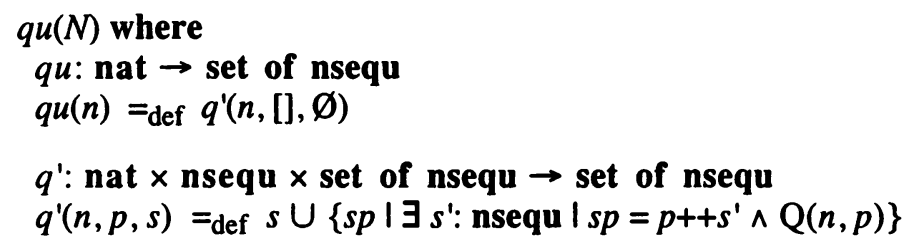

(b) Next, we aim at transforming $g^{\prime}$ as defined above. To this end, we calculate as follows:

$g^{\prime}(u, v, s)$

$=$ [ (b1) unfold $g^{\prime}$ ]

$s \cup\left\{y \mid \mathrm{Q}^{\prime}(u, v, y)\right\}$

= [ (b2) case-introduction; distributivity of $\cup$ over conditional ]

if $\mathrm{T}(u, v)$ then $s \cup\left\{y \mid \mathrm{Q}^{\prime}(u, v, y)\right\}$ else $s \cup\left\{y \mid \mathrm{Q}^{\prime}(u, v, y)\right\}$ fi

$\mathrm{T}$ is a termination condition that serves a two-fold purpose: for the very first call of $g^{\prime}$ it has to cover the "trivial case" (i.e., the case when E is already a solution for the initial input value $X$ ); for all other recursive calls of $g$ ' it has to "signal" that the incremental construction of an element of type $\mathbf{r}$ has been completed.

To be able to continue our derivation we need addditional information. To this end we require 
postulate (3) $\mathrm{P}(X, u, v) \Delta \mathrm{T}(u, v)=$ true $+\mathrm{H}(u, v)=\left\{y \mid \mathrm{Q}^{\prime}(u, v, y)\right\}$

postulate (4) $\mathrm{P}(X, u, v) \Delta \neg \mathrm{T}(u, v)=$ true $\mathrm{r}$

$\left\{y \mid Q^{\prime}(u, v, y)\right\}=$

$\left.\bigcup_{i=1 . n(u, v)}\left\{y \mid \mathrm{B}(i, u, v) \Delta \mathrm{Q}^{\prime}(\mathrm{K}(i, u, v), \mathrm{R}(i, u, v), y)\right\}\right]$

Postulate (3) gives the information how to construct the solution upon termination using an expression $\mathrm{H}$. In most applications $\mathrm{H}$ will be of the simple form $H(u, v)=\{v\}$, i.e. a singleton set.

Postulate (4) gives the information on how to split up the computation of the desired set into subcomputations the number of which, $n(u, v)$, may (dynamically) depend on the actual parameter values. The expressions $\mathbf{K}$ and $\mathbf{R}$ characterize the modification of the parameters within subcomputations.

Moreover, in order to be able to deal with the union in postulate (4) in an efficient way, we also require

postulate (5) $\operatorname{next}(k, u, v) \subseteq$

some $k^{\prime}:$ nat $\mid k^{\prime} \in\{i:$ nat $\mid k+1 \leq i \leq n(u, v)+1 \Delta$

$\forall i^{\prime}:$ nat $\left.\mid k<i^{\prime}<i \Rightarrow \neg \mathrm{B}\left(i^{\prime}, u, v\right)\right\}$

Postulate (5) describes an operation next which is characterized nondeterministically on purpose in order to be able to choose a descendant that allows pruning the computation by cutting off unsuccessful branches before they are entered. Obviously, the choice $\operatorname{next}(k, u, v)==_{\text {def }} k+1$ is a valid descendant that satisfies this postulate.

Our calculation then proceeds as follows:

- [ (b3) modification using postulate (3) and postulate (4)]

if $\mathrm{T}(u, v)$ then $s \cup \mathrm{H}(u, v)$

else $s \cup \cup_{i=1 . n(u, v)}\left\{y \mid \mathrm{B}(i, u, v) \Delta \mathrm{Q}^{\prime}(\mathrm{K}(i, u, v), \mathrm{R}(i, u, v), y)\right\} \mathrm{fi}$

- [ (b4) simplification using postulate (5)]

if $\mathrm{T}(u, v)$ then $s \cup \mathrm{H}(u, v)$

else $s \cup \bigcup_{i=n \operatorname{ext}(0, u, v) . n(u, v)}\left\{y \mid \mathrm{B}(i, u, v) \Delta \mathrm{Q}^{\prime}(\mathrm{K}(i, u, v), \mathrm{R}(i, u, v), y)\right\}$

fi

- [ (b5) abstraction ]

if $\mathrm{T}(u, v)$ then $s \cup \mathrm{H}(u, v)$ else $g^{\prime \prime}(n \operatorname{ext}(0, u, v), u, v, s)$ fi where

$\boldsymbol{g}^{\prime \prime}:(k:$ nat $\times u: \mathbf{m} \times v: \mathbf{r} \times s:$ set of $\mathbf{n} \mid \mathrm{P}(X, u, v)) \rightarrow$ set of $\mathbf{n}$

$g^{\prime \prime}(k, u, v, s)==_{\text {def }}$

$s \cup \bigcup_{i=k . n(u, v)}\left\{y \mid \mathrm{B}(i, u, v) \Delta \mathrm{Q}^{\prime}(\mathrm{K}(i, u, v), \mathrm{R}(i, u, v), y)\right\}$. 
Example (cont.):

For our running example we choose the instantiations

$$
\begin{aligned}
& \mathrm{T}(u, v) \quad \text { by } \mathrm{T}(n, p) \cong \# p=n \\
& \mathrm{H}(u, v) \quad \text { by } \mathrm{H}(n, p) \leqq\{p\} \\
& n(u, v) \quad \text { by } n(n, p) \cong n \\
& \mathrm{~B}(i, u, v) \quad \text { by } \mathrm{B}(k, n, p) \cong n c(p, k) \text { where } \\
& n c: \text { nsequ } \times \text { nat } \rightarrow \text { bool } \\
& n c(p, k)={ }_{\operatorname{def}} \forall(i \text { : nat } \mid 1 \leq i \leq \# p) \text { : } \\
& p[i] \neq k \wedge \operatorname{abs}(p[i]-k) \neq \operatorname{abs}(i-(\# p+1)) \\
& \text { (* checks whether } k \text { does not "attack" any of the elements of } p^{*} \text { ) } \\
& \mathrm{K}(i, u, v) \quad \text { by } \mathrm{K}(k, n, p) \equiv n \\
& \mathrm{R}(i, u, v) \quad \text { by } \mathrm{R}(k, n, p) \cong p++k \\
& \text { next }(k, u, v) \text { by next: nat } \times \text { nat } \times \text { nsequ } \rightarrow \text { nat } \\
& n \operatorname{ext}(k, n, p)=_{\text {def }} \min \{i: \text { nat } \mid k+1 \leq i \leq n+1 \Delta \\
& \left.\forall i^{\prime} \text { : nat } \mid k<i^{\prime}<i \Rightarrow i^{\prime} \in p\right\}
\end{aligned}
$$

and obtain as the next intermediate version

$$
\begin{aligned}
& q^{\prime}: \text { nat } \times \text { nsequ } \times \text { set of nsequ } \rightarrow \text { set of nsequ } \\
& q^{\prime}(n, p, s)=\operatorname{def} \text { if } \# p=n \text { then } s \cup\{p\} \text { else } q \text { " }(n \operatorname{ext}(0, n, p), n, p, s) \text { fi } \\
& q^{\prime \prime}: \text { nat } \times \text { nat } \times \text { nsequ } \times \text { set of nsequ } \rightarrow \text { set of nsequ } \\
& q^{\prime \prime}(k, n, p, s)=\operatorname{def} s \cup \bigcup_{i=k . n}\{s p \mid n c(p, i) \Delta \\
& \left.\quad \exists s^{\prime}: \text { nsequ } \mid s p=(p++i)++s^{\prime} \wedge \# s p=n \wedge i s p o s s e q u(s p, n) \wedge n c o n f(s p)\right\} .
\end{aligned}
$$

(c) Third, we transform $g$ " as defined in (b). We reason as follows:

$$
\begin{aligned}
& g^{\prime \prime}(k, u, v, s) \\
& \quad=[(\mathrm{c} 1) \text { unfold } g "] \\
& s \cup \bigcup_{i=k . n(u, v)}\left\{y \mid \mathrm{B}(i, u, v) \Delta \mathrm{Q}^{\prime}(\mathrm{K}(i, u, v), \mathrm{R}(i, u, v), y)\right\} \\
& =[(\mathrm{c} 2) \text { case-introduction; simplification of then-branch ] }
\end{aligned}
$$

if $k>n(u, v)$ then $s$

else $s \cup \bigcup_{i=k . n(u, v)}\left\{y \mid \mathrm{B}(i, u, v) \Delta \mathrm{Q}^{\prime}(\mathrm{K}(i, u, v), \mathrm{R}(i, u, v), y)\right\}$ fi.

For the else-branch we reason as follows:

$$
\begin{aligned}
& s \cup \bigcup_{i=k . n(u, v)}\left\{y \mid \mathrm{B}(i, u, v) \Delta \mathrm{Q}^{\prime}(\mathrm{K}(i, u, v), \mathrm{R}(i, u, v), y)\right\} \\
& \equiv[(\mathrm{c} 3) \cup \text {-split in else-branch ] } \\
& s \cup\left\{y \mid \mathrm{B}(k, u, v) \Delta \mathrm{Q}^{\prime}(\mathrm{K}(k, u, v), \mathrm{R}(k, u, v), y)\right\} \cup \\
& \bigcup_{i=k+1 . . n e x t(k, u, v)-1}\left\{y \mid \mathrm{B}(i, u, v) \Delta \mathrm{Q}^{\prime}(\mathrm{K}(i, u, v), \mathrm{R}(i, u, v), y)\right\} \cup
\end{aligned}
$$




$$
\bigcup_{i=n \operatorname{ext}(k, u, v) . . n(u, v)}\left\{y \mid \mathrm{B}(i, u, v) \Delta \mathrm{Q}^{\prime}(\mathrm{K}(i, u, v), \mathrm{R}(i, u, v), y)\right\}
$$

$=\left[\right.$ (c4) simplification: def. next $\left.\Rightarrow \bigcup_{i=k+1 . \text { next }(k, u, v)-1}\{\ldots\} \equiv \emptyset\right]$

$s \cup\left\{y \mid \mathrm{B}(k, u, v) \Delta \mathrm{Q}^{\prime}(\mathrm{K}(k, u, v), \mathrm{R}(k, u, v), y)\right\} \cup$

$\bigcup_{i=n \operatorname{ext}(k, u, v) . . n(u, v)}\left\{y \mid \mathrm{B}(i, u, v) \Delta \mathrm{Q}^{\prime}(\mathrm{K}(i, u, v), \mathrm{R}(i, u, v), y)\right\}$

$=$ [ (c5) case introduction; simplification ]

if $\mathrm{B}(k, u, v)$

then $s \cup\left\{y \mid \mathrm{Q}^{\prime}(\mathrm{K}(k, u, v), \mathrm{R}(k, u, v), y)\right\} \cup$

$$
\bigcup_{i=\operatorname{next}(k, u, v) . n(u, v)}\left\{y \mid \mathrm{B}(i, u, v) \Delta \mathrm{Q}^{\prime}(\mathrm{K}(i, u, v), \mathrm{R}(i, u, v), y)\right\}
$$

else $s \cup \bigcup_{i=n \operatorname{ext}(k, u, v) . . n(u, v)}\left\{y \mid \mathrm{B}(i, u, v) \Delta \mathrm{Q}^{\prime}(\mathrm{K}(i, u, v), \mathrm{R}(i, u, v), y)\right\} \mathbf{f i}$

Our next steps aim at folding $g^{\prime}$ and $g^{\prime \prime}$. Since both functions have the same invariant $P$, we have to make sure that the invariant is maintained. To this end, we require

postulate (6) $1 \leq i \leq n(u, v) \Delta \mathrm{P}(X, u, v) \Delta \neg \mathrm{T}(u, v) \Delta \mathrm{B}(i, u, v) \equiv$ true $\mathrm{P}(X, \mathrm{~K}(i, u, v), \mathrm{R}(i, u, v))=$ true

Our calculation then continues as follows

= [ (c6) fold $g^{\prime}$ - postulate (6) guarantees assertion; termination below] if $\mathrm{B}(k, u, v)$

then $g^{\prime}(\mathrm{K}(k, u, v), \mathrm{R}(k, u, v), s) \cup$

$\bigcup_{i=n \operatorname{ext}(k, u, v) . . n(u, v)}\left\{y \mid \mathrm{B}(i, u, v) \Delta \mathrm{Q}^{\prime}(\mathrm{K}(i, u, v), \mathrm{R}(i, u, v), y)\right\}$

else $s \cup \cup_{i=n \operatorname{ext}(k, u, v) . . n(u, v)}\left\{y \mid \mathrm{B}(i, u, v) \Delta \mathrm{Q}^{\prime}(\mathrm{K}(i, u, v), \mathrm{R}(i, u, v), y)\right\} \mathrm{fi}$

$=$ [ (c7) fold $g$ " - assertion remains valid; termination below]

if $\mathrm{B}(k, u, v)$ then $g^{\prime \prime}\left(n \operatorname{ext}(k, u, v), u, v, g^{\prime}(\mathrm{K}(k, u, v), \mathrm{R}(k, u, v), s)\right)$

else $g^{\prime \prime}(\operatorname{next}(k, u, v), u, v, s)$ fi.

Altogether, we have:

$g^{\prime \prime}(k, u, v, s)=_{\text {def }}$

if $k>n(u, v)$ then $s$

elsf $\mathrm{B}(k, u, v)$ then $g^{\prime \prime}\left(\operatorname{next}(k, u, v), u, v, g^{\prime}(\mathrm{K}(k, u, v), \mathrm{R}(k, u, v), s)\right)$

else $g^{\prime \prime}(n \operatorname{ext}(k, u, v), u, v, s)$ fi 
Example (cont.)

Applying this to our running example we yield

$$
\begin{aligned}
& q^{\prime \prime}: \text { nat } \times \text { nat } \times \text { nsequ } \times \text { set of nsequ } \rightarrow \text { set of nsequ } \\
& q^{\prime \prime}(k, n, p, s)=\operatorname{def} \\
& \quad \text { if } k>n \text { then } s \\
& \text { elsf } n c(p, k) \text { then } q^{\prime \prime}\left(n e x t(k, n, p), n, p, q^{\prime}(n, p++k, s)\right) \\
& \quad \text { else } q^{\prime \prime}(n e x t(k, n, p), n, p, s) \text { fi. }
\end{aligned}
$$

(d) It remains to prove termination of $g^{\prime}$ and $g$ " which is required for the correctness of the folding steps. By our general convention, all expressions are defined. In the result obtained so far, $g^{\prime}$ can be eliminated by unfolding its calls in $g$ and g":

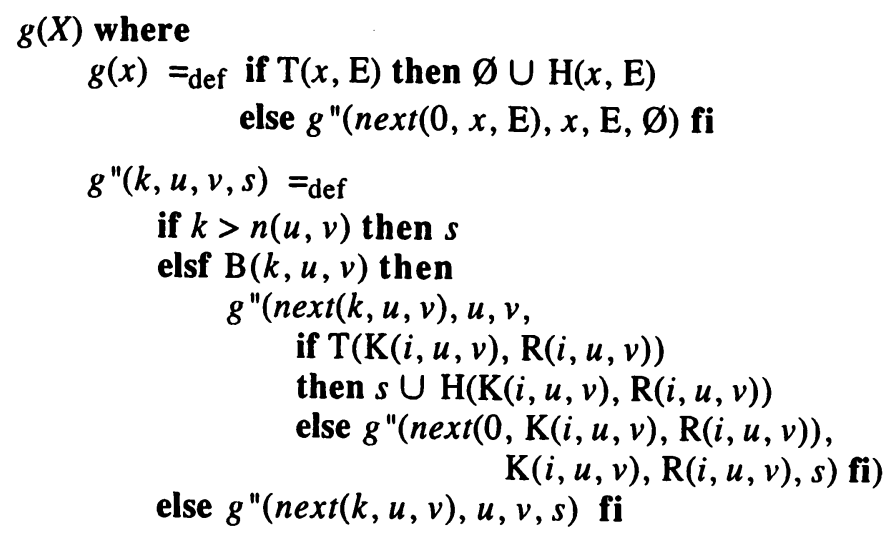

Simplification and distributivity of function call over conditional (in $g "$ ) then yields

$$
\begin{aligned}
& g^{\prime \prime}(k, u, v, s)==_{\mathrm{def}} \\
& \text { if } k>n(u, v) \text { then } s \\
& \text { elsf } \mathrm{B}(k, u, v) \text { then } \\
& \text { if } \mathrm{T}(\mathrm{K}(i, u, v), \mathrm{R}(i, u, v)) \\
& \text { then } g^{\prime \prime}(\operatorname{next}(k, u, v), u, v, s \cup \mathrm{H}(\mathrm{K}(i, u, v), \mathrm{R}(i, u, v))) \\
& \text { else } g^{\prime \prime}\left(n \operatorname{ext}(k, u, v), u, v, g^{\prime \prime}(\operatorname{next}(0, \mathrm{~K}(i, u, v), \mathrm{R}(i, u, v)),\right. \\
& \text { else } g^{\prime \prime}(n \operatorname{ext}(k, u, v), u, v, s) \mathrm{fi}
\end{aligned}
$$

Termination of $g$ is obvious provided $g$ " terminates. To prove termination of $g$ " we define the following (lexicographic) termination ordering $\angle$ on nat $\times \mathbf{m} \times \mathbf{r}$ $x$ set of $n$ :

$$
\begin{aligned}
& (k, u, v, s)<\left(k^{\prime}, u^{\prime}, v^{\prime}, s^{\prime}\right) \Leftrightarrow \quad v<v^{\prime} \vee\left(v=v^{\prime} \wedge n(u, v)-k<n(u, v)-k^{\prime}\right) \\
& \vee \quad u<u^{\prime} \vee\left(u=u^{\prime} \wedge n(u, v)-k<n(u, v)-k^{\prime}\right)
\end{aligned}
$$


which is well-defined, if we have a well-founded ordering $<$ either on $\mathbf{m}$ or $\mathbf{r}$. Therefore we require

$$
\begin{aligned}
\text { postulate (7) } 1 \leq i \leq n(u, v) \Delta \mathrm{B}(i, u, v)=\text { true t } \\
(\mathrm{R}(i, u, v)<v)=\text { true where } \mathrm{WF}-\mathrm{ORD}(\mathbf{r},<) \text { or } \\
(\mathrm{K}(i, u, v)<u)=\text { true where WF-ORD }(\mathrm{m},<)
\end{aligned}
$$

Then, for the recursive calls of $g$ " we have

$$
\begin{aligned}
& (n \operatorname{ext}(k, u, v), u, v, s \cup \mathrm{H}(\mathrm{K}(i, u, v), \mathrm{R}(i, u, v)))<(k, u, v, s) \\
& \text { [ by def. of } \angle \text { next ] } \\
& (\operatorname{next}(0, \mathrm{~K}(i, u, v), \mathrm{R}(i, u, v)), \mathrm{K}(i, u, v), \mathrm{R}(i, u, v), s) \angle(k, u, v, s) \\
& \left(n \operatorname{ext}(k, u, v), u, v, g^{\prime \prime}(\operatorname{next}(0, \mathrm{~K}(i, u, v), \mathrm{R}(i, u, v)) \text {, }\right. \\
& \text { [ by postulate (7) ] } \\
& \mathrm{K}(i, u, v), \mathrm{R}(i, u, v), s))<(k, u, v, s) \\
& (\operatorname{next}(k, u, v), u, v, s)<(k, u, v, s) \\
& \text { [ by def. of } \angle \text { and next ] } \\
& \text { [ by def. of } \angle \text { and next ] }
\end{aligned}
$$

Hence, $g$ " terminates.

Example (cont.):

For the proof of termination, we refer the reader to Sect. 3.1, where the solution of the N-Queens problem is completed.

\subsection{The rule}

Obviously, the derivation given in Sect. 2.1 is a (constructive) proof of a transformation rule composed of

- the initial specifiction as input scheme

- the result of the derivation as output scheme

- the set of postulates as applicability conditions.

Additionally, the derivation above can be slightly generalized to also cover specifications of the form

$$
f(x)={ }_{\operatorname{def}}\{r(X, y): \mathbf{n} \mid \mathrm{Q}(x, y)\}
$$

(where ZF set abstraction is used as an abbreviation for $\{q: \mathbf{n}|\exists y: \mathbf{p}| q=r(X, y)$ $\Delta \mathrm{Q}(x, y)\})$, since the substitution of $y$ by $r(X, y)$ does not invalidate our derivation.

With this generalization our rule reads: 
$f(X)$ where

$f(x)=_{\operatorname{def}}\{r(X, y): \mathbf{n} \mid \mathrm{Q}(x, y)\}$

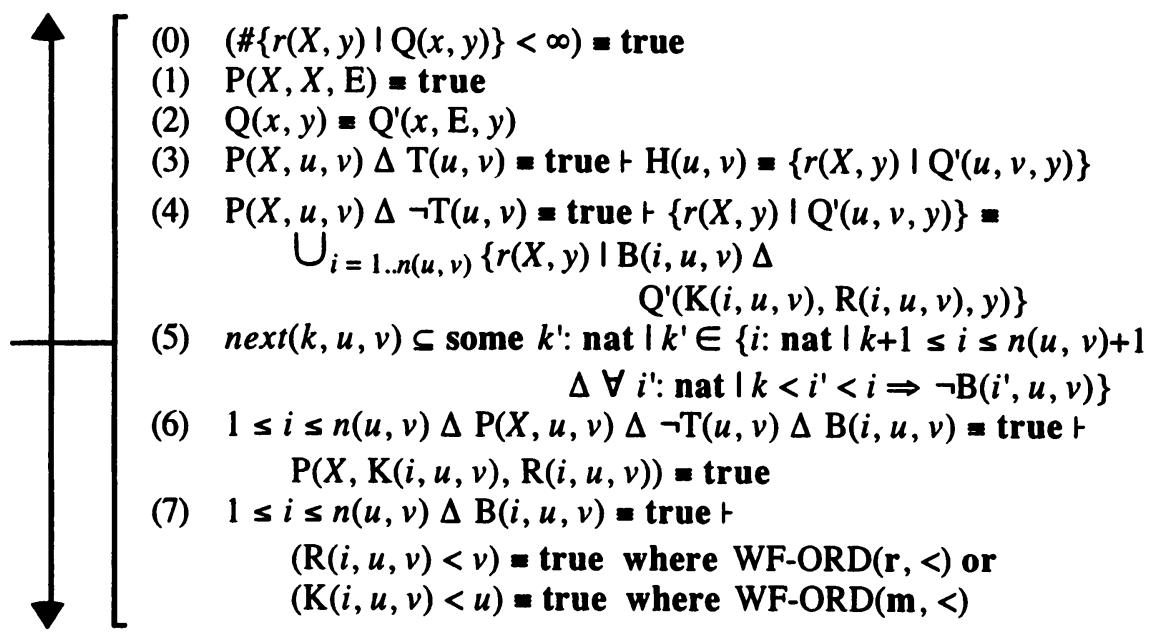

$g(X)$ where

$g(x)={ }_{\operatorname{def}} g^{\prime}(x, \mathrm{E}, \varnothing)$

$g^{\prime}(u, v, s)==_{\text {def }}$

if $\mathrm{T}(u, v)$ then $s \cup \mathrm{H}(u, v)$ else $g^{\prime \prime}(\operatorname{next}(0, u, v), u, v, s)$ fi

$g^{\prime \prime}(k, u, v, s)==_{\operatorname{def}}$

if $k>n(u, v)$ then $s$

elsf $\mathrm{B}(k, u, v)$ then $g$ " $\left(n \operatorname{ext}(k, u, v), u, v, g^{\prime}(\mathrm{K}(k, u, v), \mathrm{R}(k, u, v), s)\right)$

else $g^{\prime \prime}(n \operatorname{ext}(k, u, v), u, v, s)$ fi

\section{Syntactic constraints}

$\mathrm{KIND}[r]=\mathbf{m} \times \mathbf{p} \rightarrow \mathbf{n}$

$\operatorname{KIND}[f, g]=\mathbf{m} \rightarrow$ set of $\mathbf{n}$

$\operatorname{KIND}\left[g^{\prime}\right]=(u: \mathbf{m} \times v: \mathbf{r} \times s:$ set of $\mathbf{n} \mid \mathrm{P}(X, u, v)) \rightarrow$ set of $\mathbf{n}$

$\mathrm{KIND}\left[g^{\prime \prime}\right]=(k:$ nat $\times u: \mathbf{m} \times v: \mathbf{r} \times s:$ set of $\mathbf{n} \mid \mathrm{P}(X, u, v)) \rightarrow$ set of $\mathbf{n}$

$\mathrm{KIND}[$ next $]=($ nat $\times \mathbf{m} \times \mathbf{r}) \rightarrow$ nat

Admittedly, the whole bunch of applicability conditions looks complicated at a first glance, due to the many expressions to be "invented" and the lot of resulting proof obligations. As to the first, one should keep in mind that in most applications the simple forms as commented above are sufficient - which substantially reduces the amount of work for finding suitable instantiations. As to the latter, it will be examplified with the queens example below, that, whenever suitable instantiations have been found, all proofs of the instantiated applicability conditions are straightforward or even trivial. 


\section{SOME APPLICATIONS OF THE RULE}

In this section we complete the treatment of the queens example and deal with another (non-trivial) problem to illustrate the power of our rule and how to use it.

\subsection{The N-Queens Problem completed}

In summary, the application of our rule to the queens problem (with $r$ instantiated by the identity on its second argument, and WF-ORD $(\mathbf{r},<)$ instantiated by (nsequ, $a<b \Leftrightarrow n-\# a<n-\# b)$ ) results in the following program:

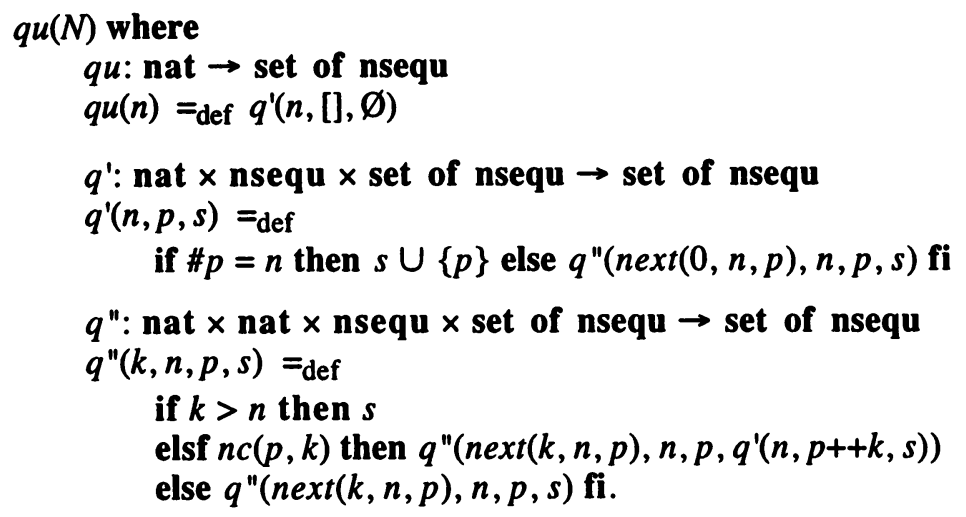

It remains to prove the instantiated applicability conditions (which is straightforward or even trivial):

(0) $(\#\{s p:$ nsequ I \#sp $=n \wedge$ ispossequ $(s p, n) \wedge n \operatorname{conf}(s p)\}<\infty) \equiv$ true

Trivial, as already nsequ restricted by ispossequ has only finitely many elements (for any $n$ ).

(1) $(\#[] \leq n \wedge$ ispossequ $([], n) \wedge n \operatorname{conf}([]))=$ true

Obviously, $\#[]=0 \leq n$, ispossequ $([], n)$, and $n \operatorname{conf}([])$ are all true.

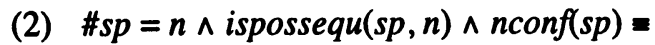

$$
\exists s^{\prime}: \mathrm{nsequ} \mid s p=[]++s^{\prime} \wedge \# s p=n \wedge i \operatorname{ispossequ}(s p, n) \wedge n \operatorname{conf}(s p)
$$

Trivial, as for $s^{\prime}=s p$ the righthand side expression simplifies to the one on the lefthand side.

(3) $(\# p \leq n \wedge$ ispossequ $(p, n) \wedge n \operatorname{conf}(p)) \Delta \# p=n=\operatorname{true} \vdash$

$$
\begin{aligned}
\{p\}=\left\{s p: \mathrm{nsequ}\left|\exists s^{\prime}: \mathrm{nsequ}\right| s p=p++s^{\prime} \wedge \# s p\right. & =n \wedge \\
i \operatorname{ispossequ}(s p, n) & \wedge n \operatorname{nconf}(s p)\}
\end{aligned}
$$


From $\# p=n, \# s p=n$, and $s p=p++s^{\prime}$, it follows that $\# s^{\prime}=0$, and, thus, $s^{\prime}=$ []. Hence, the set on the righthand side of the consequence simplifies to

$\{s p:$ nsequ $\mid s p=p \wedge \# s p=n \wedge$ ispossequ $(s p, n) \wedge n \operatorname{conf}(s p)\}$.

The equality of both sets then follows from the premise on $p$.

(4) (\#p $\leq n \wedge$ ispossequ $(p, n) \wedge n \operatorname{conf}(p)) \Delta \# p<n \equiv$ true ト

$\left\{s p:\right.$ nsequ $\mid \exists s^{\prime}:$ nsequ $\mid s p=p++s^{\prime} \wedge \# s p=n \wedge$

$$
\begin{array}{r}
\text { ispossequ }(s p, n) \wedge n \operatorname{conf}(s p)\}= \\
\bigcup_{i=1 . . n}\left\{s p: \text { nsequ }\left|n c(p, i) \Delta \exists s^{\prime}: \operatorname{nsequ}\right| s p=(p++i)++s^{\prime} \wedge\right. \\
\# s p=n \wedge i \operatorname{spossequ}(s p, n) \wedge n \operatorname{conf}(s p)\}
\end{array}
$$

Due to the premise \#p<n and associativity of ++ , the lefthand side of the consequence can be rewritten into

$\left\{s p:\right.$ nsequ $\mid \exists s^{\prime}:$ nsequ, $i: \operatorname{pos}(n) \mid s p=(p++i)++s^{\prime} \wedge$

$$
\# s p=n \wedge \operatorname{ispossequ}(s p, n) \wedge n \operatorname{conf}(s p)\}
$$

where $i: \operatorname{pos}(n)$ abbreviates ( $i$ : nat $\mid 1 \leq i \leq n)$.

Since $\neg n c(p, i)$ implies $\neg n c o n f\left((p++i)++s^{\prime}\right)$, the existential quantification on $i$ can be restricted to those $i$ that satisfy $n c(p, i)$. The requested equality then follows from the general rule

$$
\{x|\exists y, z| \mathrm{P}(z) \wedge \mathrm{Q}(x, y, z)\}=\bigcup_{z \mid \mathrm{P}(z)}\{x|\exists y| \mathrm{Q}(x, y, z)\}
$$

(5) $\min \left\{i\right.$ : nat $\mid k+1 \leq i \leq n+1 \Delta \forall i^{\prime}:$ nat $\left.\mid k<i^{\prime}<i \Rightarrow i^{\prime} \in p\right\} \subseteq$ some $k^{\prime}:$ nat $\mid k^{\prime} \in\left\{i\right.$ : nat $\mid k+1 \leq i \leq n+1 \Delta \forall i^{\prime}:$ nat $\mid$

$$
\left.k<i^{\prime}<i \Rightarrow \neg n c\left(p, i^{\prime}\right)\right\}
$$

Obviously, $i^{\prime} \in p$ implies $\neg n c\left(p, i^{\prime}\right) . \min m$ is always a descendant of some $x \mid x \in m$.

(6) $1 \leq i \leq n \Delta(\# p \leq n \wedge i \operatorname{spossequ}(p, n) \wedge n \operatorname{conf}(p)) \Delta \# p<n \Delta n c(p, i)=$ true $\vdash \#(p++i) \leq n \wedge$ ispossequ $(p++i, n) \wedge n \operatorname{conf}(p++i) \equiv$ true

$\# p<n$ implies \# $(p++i) \leq n$, ispossequ $(p++i, n)$ follows immediately from the premise, and $n c o n f(p++i)$ follows from $n c o n f(p)$ and $n c(p, i)$.

(7) $1 \leq i \leq n \Delta n c(p, i)=$ true $\vdash n-\#(p++i)<n-\# p=$ true

The consequence is even true for arbitrary $n, p$, and $i$. 


\section{Remarks}

- The choice of next as defined above (instead of the generally possible choice $n \operatorname{ext}(k)=\operatorname{def} k+1)$ is a simple optimization (according to the remark on next in Sect. 2).

- As a further improvement, we could have added another parameter to keep all possible candidate positions to be added to the current sequence. This would require modified instantiations, viz.

$$
\begin{array}{lll}
\mathrm{P}(X, u, v) & \text { by } \# p \leq n \wedge i \operatorname{spossequ}(p, n) \wedge \underset{n c o n f(p) \wedge}{\forall(i: \operatorname{pos}(n) \mid i \in c) \mid n c(p, i)} \\
\mathrm{E} & \text { by }([],\{i: \operatorname{pos}(n)\}) \\
\mathrm{B}(i, u, v) & \text { by } c \neq \varnothing \\
\mathrm{R}(i, u, v) & \text { by }(p++i,\{k: \operatorname{pos}(n) \mid n c(p++i, k)\}) .
\end{array}
$$

\section{$\forall(i: \operatorname{pos}(n) \mid i \in c) \mid n c(p, i)$}

Together with

$$
n \operatorname{ext}(k, c)=_{\operatorname{def}} \text { if } c \neq \varnothing \text { then } \min c \text { else } n+1 \mathrm{fi}
$$

these modified instantiations then would have resulted in

$$
\begin{aligned}
& q u(N) \text { where } \\
& q u(n)={ }_{\operatorname{def}} q^{\prime}(n,[],\{i: \operatorname{pos}(n)\}, \varnothing) \\
& q^{\prime}(n, p, c, s)==_{\text {def }} \\
& \text { if \#p=n then } s \cup\{p\} \text { else } q^{\prime \prime}(n \operatorname{ext}(0, c), n, p, c, s) \text { fi } \\
& q^{\prime \prime}(k, n, p, c, s)=_{\mathrm{def}} \\
& \text { if } k>n \text { then } s \\
& \text { elsf } c \neq \varnothing \text { then } \\
& \left.q^{\prime \prime}\left(\operatorname{next}(k, c), n, p, c, q^{\prime}(n, p++k,\{i: \operatorname{pos}(n)\} \mid n c(p++k, i)\}, s\right)\right) \\
& \text { else } q . "(n \operatorname{ext}(k, c), n, p, c, s) \text { fi. }
\end{aligned}
$$

As a further improvement - which is left to the interested reader - one could think of applying finite differencing to efficiently compute the new candidate set in the first recursive call of $g$ ".

\subsection{A Prolog Interpreter}

A Prolog interpreter takes a Prolog program and a query and yields as a result either the set of all substitutions (for the variables in the query) such that the instantiated query is a logical consequence from the program, or it does not terminate. If there is no substitution (such that the instantiated query follows from the program) the result is the empty set.

To formally specify the problem, we first have to define the notions of program, query, and substitution. A program is a sequence of clauses (or rules) each of which consists of a literal as its lefthand side and a (possibly empty) sequence of literals as its righthand side. A literal in turn consists of a predicate symbol and a (possibly 
empty) sequence of terms (as arguments). And a term is either a constant symbol, a variable symbol, or a function symbol together with a sequence of terms as arguments. All this can straightforwardly be formalized as follows:

$$
\begin{aligned}
& \text { program }=_{\text {def }} \text { sequ of clause } \\
& \text { clause }=_{\text {def }}(l h s: \text { literal, } r h s: \text { sequ of literal) } \\
& \text { literal }=_{\text {def }}(p s: \text { predsymb, st: sequ of term) } \\
& \text { term }=_{\text {def }} \text { const } \mid \text { var } \mid \text { (functsymb, sequ of term). }
\end{aligned}
$$

A query is a sequence of literals, and a substitution is an association of variable symbols with terms. Again, formalization is straightforward:

$$
\begin{aligned}
& \text { query }=\text { def sequ of literal } \\
& \text { subst }={ }_{\text {def }} \text { EMAP(var, term, =). }
\end{aligned}
$$

To construct a substitution and simultaneously to prove that the instantiated query $g l$ follows from the program $p$, a prolog interpreter uses sld-resolution which is captured by the following derivability relation:

$$
\begin{aligned}
& \vec{p}: \text { query } \times \text { subst } \times \text { query } \times \text { subst } \rightarrow \text { bool } \\
& (g l, \Theta) \vec{p}\left(g l^{\prime}, \Theta \circ \Theta^{\prime}\right)={ }_{\text {def }} \\
& \exists c \in p \mid g l^{\prime}=\left(r h s\left(c^{\prime}\right)++ \text { tl } g l\right) \Theta^{\prime} \wedge \\
& c^{\prime}=r n(c, g l) \wedge \text { unify }\left(\text { hd } g l, \operatorname{lh} s\left(c^{\prime}\right)\right) \neq \text { fail } \Delta \\
& \Theta^{\prime}=\text { unify }\left(\text { hd } g l, \operatorname{lhs}\left(c^{\prime}\right)\right)
\end{aligned}
$$

where

$\Theta \cdot \Theta^{\prime}$ denotes the composition of the substitutions $\Theta$ and $\Theta^{\prime}$,

$a \Theta$ denotes the application of the substitution $\Theta$ to $a$,

$r n$ : clause $\times$ query $\rightarrow$ clause

$r n(c, g l)==_{\text {def }}$

« clause $c$ with all variables renamed such that they do not occur in $g l$ »

and (with $c_{i}$ and $v_{i}$ denoting constant and variable symbols, respectively, $f, g$ denoting function symbols, and $r_{i}, s_{i}$ denoting terms)

$$
\begin{aligned}
& \text { struct }=\text { def term I literal } \\
& \text { unify: struct } \times \text { struct } \rightarrow \text { subst I fail } \\
& \text { unify }\left(c_{1}, c_{2}\right)={ }_{\text {def }} \text { if } c_{1}=c_{2} \text { then } \emptyset \text { else fail fi } \\
& \text { unify }\left(c_{1}, v_{2}\right)==_{\operatorname{def}}\left(c_{1} \text { for } v_{2}\right) \\
& \text { unify }\left(c_{1}, g\left(r_{1}, \ldots, r_{k}\right)\right)=\text { def fail } \\
& \text { unify }\left(v_{1}, c_{2}\right)==_{\operatorname{def}}\left(c_{2} \text { for } v_{1}\right) \\
& \text { unify }\left(v_{1}, v_{2}\right)==_{\operatorname{def}} \text { if } v_{1} \neq v_{2} \text { then }\left(v_{2} \text { for } v_{1}\right) \text { else } \varnothing \mathbf{f i}
\end{aligned}
$$




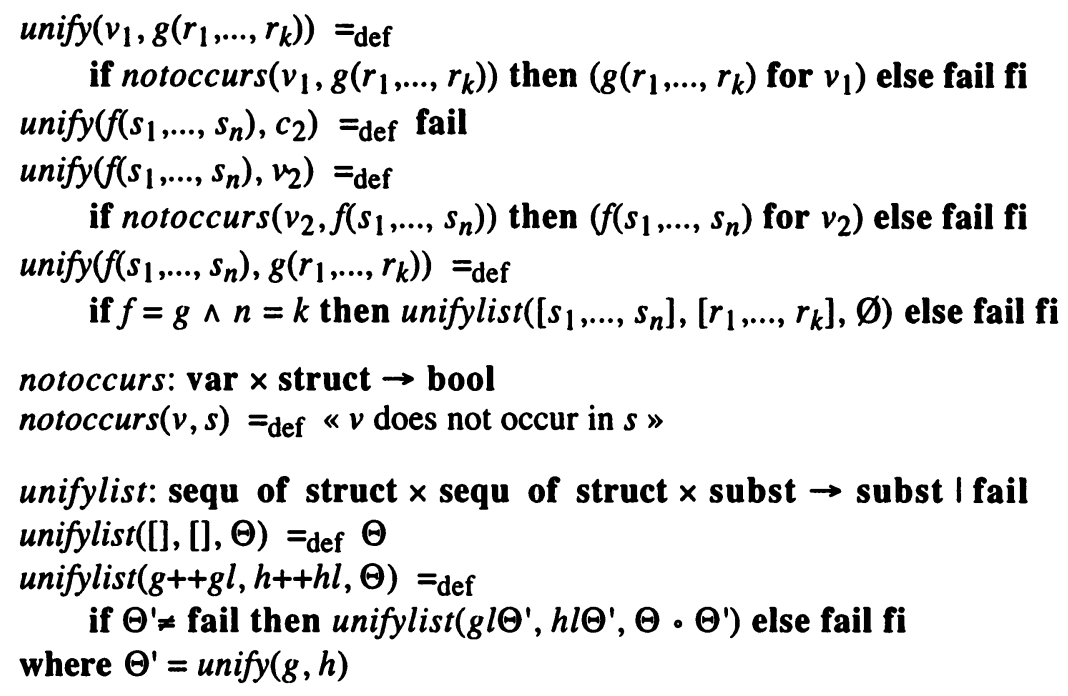

Equipped with this environment, a formal specification of a prolog interpreter is as follows:

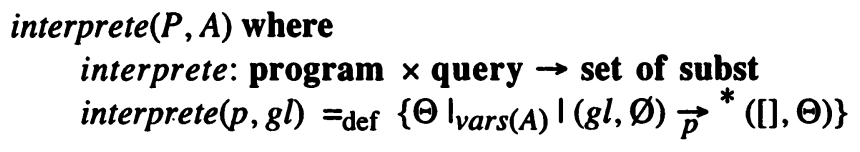

where

$\Theta I_{\text {vars }(A)}$ denotes $\Theta$ restricted to those variable symbols occurring in $A$ and

$\vec{p}^{*}$ denotes the reflexive transitive closure of $\vec{p}$.

Correct application of our transformation rule requires to satisfy the applicability condition

$$
\left(\#\left\{\left.\Theta\right|_{\operatorname{vars}(A)} \mid(g l, \varnothing) \vec{p}^{*}([], \Theta)\right\}<\infty\right)=\text { true. }
$$

Whereas in the previous example, the corresponding proof obligation was straightforward to be shown, it is less trivial here. Indeed, it only can be proved if both the program and the query satisfy additional constraints the detailed treatment of which is beyond the scope of this paper. A similar remark holds for condition (7) about the existence of a suitable well-founded ordering. Therefore we simply take these applicability conditions for granted and yield as result of rule application (as to the respective instantiations, cf. (Achatz, Partsch, 1996)): 


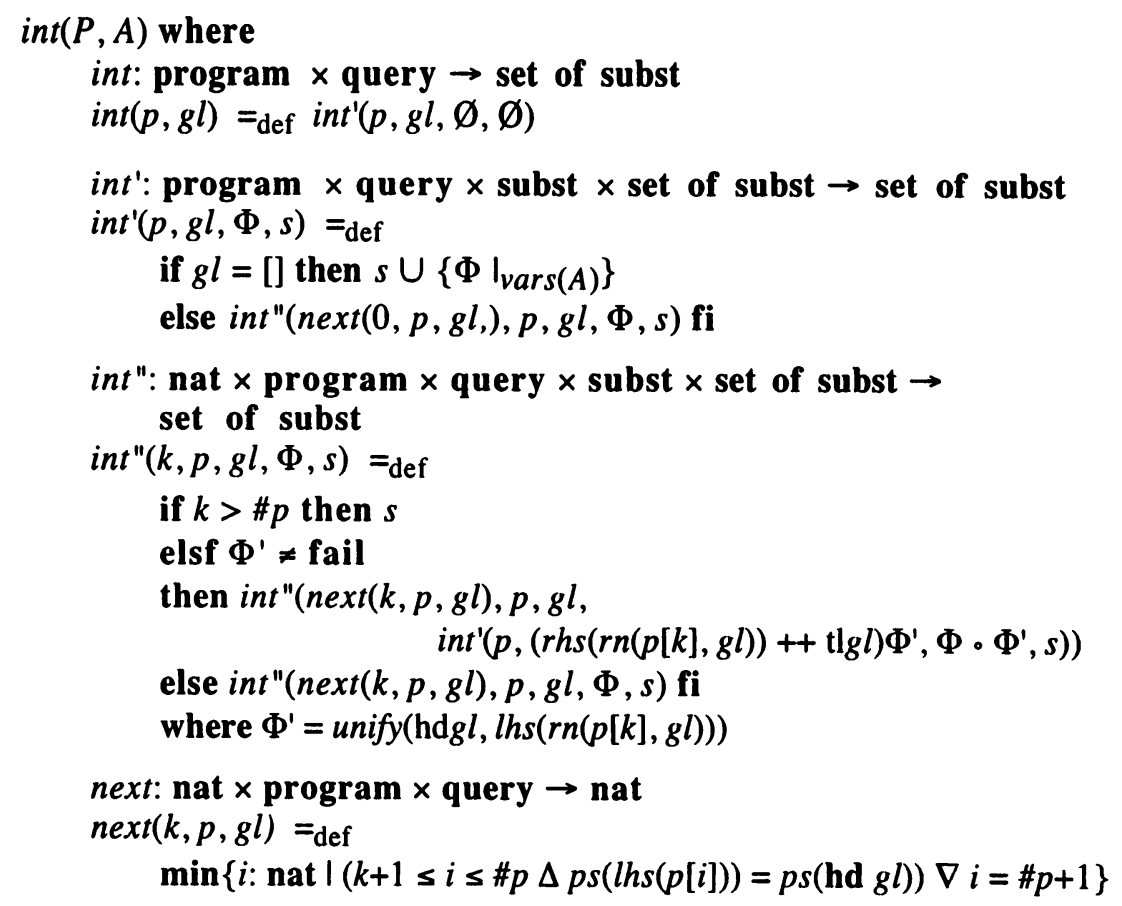

\subsection{Further examples}

Of course, there are many more problems our rule might be applied to. Apart from the additional ones dealt with in (Achatz, Partsch, 1996) (i.e., all permutations of a sequence, all partitions of a natural number, all subsequences of a sequence, a knapsack-problem, all topological sortings, all maximal, cycle-free paths from a given node), these range, for example, from mathematical problems (e.g, all subsets of a set, all partitions of a set, all subsets of a set up to a fixed size), combinatorial problems (e.g., space-filling puzzles, other known problems on the chessboard), or further graph problems.

\section{VARIANTS OF THE GENERAL RULE AND THEIR APPLICATIONS}

In the following we discuss a couple of "variants" of our general rule and illustrate them by respective examples. These "variants" look very similar to our general rule, although they deal with different kinds of specifications and require (slightly) different applicability conditions. Using the word "variant" is simply motivated by the fact that the proofs of these rules only marginally deviate from the proof of our general rule. 


\subsection{Variant 1: Existential quantification}

\subsubsection{The rule}

$f(X)$ where

$$
f(x)==_{\text {def }} \exists y: \mathbf{p} \mid \mathrm{Q}(x, y)
$$

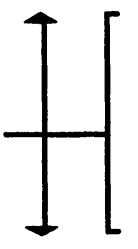

(1), (2), (5), (6), (7) as in general rule

(3) $\mathrm{P}(X, u, v) \Delta \mathrm{T}(u, v) \equiv$ true t true $=\exists y: \mathrm{p} \mid \mathrm{Q}^{\prime}(u, v, y)$

(4) $\mathrm{P}(X, u, v) \Delta \neg \mathrm{T}(u, v)=$ true $\mathrm{t}$

$\exists y: \mathbf{p} \mid \mathrm{Q}^{\prime}(u, v, y)=$

$$
\exists_{i=1 . n(u, v)} \exists y: \mathrm{p} \mid \mathrm{B}(i, u, v) \Delta \mathrm{Q}^{\prime}(\mathrm{K}(i, u, v), \mathrm{R}(i, u, v), y)
$$

$g(X)$ where

$g(x)={ }_{\text {def }} g^{\prime}(x, \mathrm{E}$, false $)$

$g^{\prime}(u, v, s)==_{\text {def }}$ if $\mathrm{T}(u, v)$ then true else $g^{\prime \prime}(\operatorname{next}(0, u, v), u, v, s) \mathrm{fi}$

$g^{\prime \prime}(k, u, v, s)==_{\operatorname{def}}$

if $s \vee k>n(u, v)$ then $s$

elsf $\mathrm{B}(k, u, v)$ then $g^{\prime \prime}\left(n \operatorname{ext}(k, u, v), u, v, g^{\prime}(\mathrm{K}(k, u, v), \mathrm{R}(k, u, v), s)\right)$ else $g$ " $(n \operatorname{ext}(k, u, v), u, v, s) \mathrm{fi}$

\section{Syntactic constraints}

$\mathrm{KIND}[f, g]=\mathbf{m} \rightarrow$ bool

$\mathrm{KIND}\left[g^{\prime}\right]=(u: \mathrm{m} \times v: \mathbf{r} \times s:$ bool I $\mathrm{P}(X, u, v)) \rightarrow$ bool

$\mathrm{KIND}\left[g^{\prime \prime}\right]=(k:$ nat $\times u: \mathrm{m} \times v: \mathbf{r} \times s:$ bool $\mathrm{P}(X, u, v)) \rightarrow$ bool

$\mathrm{KIND}[$ next $]=($ nat $\times \mathbf{m} \times \mathbf{r}) \rightarrow$ nat

\section{Proof (changes)}

a) (a2'): unfold $g^{\prime}$; neutrality of false w.r.t. $v$

b) (b2'): case-introduction; distributivity $\vee$ over conditional

c) (c3'): v-split in else-branch

4.1.2 An Application: Top-down recognition (for context-free grammars) Given a context-free grammar $G=(N, T, Z, P)$ (where $N$ is a non-empty set of nonterminal symbols, $T$ a non-emtpy set of terminal symbols which is disjoint from $N, Z \in N$, and $P$ is a non-empty set of context-free productions consisting of a nonterminal symbol as its lefthand side and a (possibly empty) sequence of (nonterminal or terminal) symbols as its righthand side) and a terminal word $w$, it is requested to check whether $w$ is derivable from $Z$ using $P$. 
Using

$$
\begin{aligned}
& \text { nont }==_{\text {def }} \text { "nonterminal symbols } \\
& \text { term }={ }_{\text {def }} \text { "terminal symbols } \\
& \text { symb }==_{\text {def }} \text { nont } 1 \text { term } \\
& \text { prod }=_{\text {def }} \text { (lhs: nont, rhs: sequ of symb) }
\end{aligned}
$$

the problem can be formalized by

$$
\begin{aligned}
& \text { recognize }(P, W) \text { where } \\
& \text { recognize: sequ of prod } \times \text { sequ of term } \rightarrow \text { bool }
\end{aligned}
$$$$
\text { recognize }(p, w)==_{\text {def }} \exists y \text { : sequ of term } \mid[Z] \vec{p}^{*} y \wedge y=w
$$

where

$$
\begin{aligned}
& \vec{p}: \text { sequ of symb } \times \text { sequ of symb } \rightarrow \text { bool } \\
& x \vec{p} y=\text { def } \exists l, r: \text { sequ of symb, } i: \text { nat } 1 \\
& x=l++l h s(p[i])++r \wedge y=l++r h s(p[i])++r
\end{aligned}
$$

and

$$
\vec{p}^{*} \text { denotes the reflexive transitive closure of } \vec{p} \text {. }
$$

The result of rule application is

$$
\begin{aligned}
& \operatorname{rec}(P, W) \text { where } \\
& \text { rec: sequ of prod } \times \text { sequ of term } \rightarrow \text { bool } \\
& \operatorname{rec}(p, w)=_{\operatorname{def}} \operatorname{rec}^{\prime}(p, w,[Z], \text { false }) \\
& \text { rec': sequ of prod } \times \text { sequ of term } \times \text { sequ of } 5 y m b \times \text { bool } \rightarrow \\
& \text { bool } \\
& \operatorname{rec}^{\prime}(p, w, v, s)=_{\text {def }} \\
& \text { if } w=[] \wedge v=[] \text { then true else } \operatorname{rec} "(\operatorname{next}(1, p, v), p, w, v, s) \text { fi } \\
& \text { rec": nat } \times \text { sequ of prod } \times \text { sequ of term } \times \text { sequ of symb } \times \text { bool } \\
& \rightarrow \text { bool } \\
& \operatorname{rec}^{\prime \prime}(k, p, w, v, s)=_{\text {def }} \\
& \text { if } s \vee k>\# p+1 \text { then } s \\
& \text { elsf }(k \leq \# p \wedge v \neq[] \Delta h \mathrm{~h} v=\operatorname{lhs}(p[k])) \vee \\
& (k=\# p+1 \wedge w \neq[] \wedge v \neq[] \Delta \mathbf{h d} w=\mathbf{h d} v) \\
& \text { then } \operatorname{rec}^{\prime \prime}(\operatorname{next}(k, p, v), p, w, v \text {, } \\
& \operatorname{rec}^{\prime}(p, \text { if } k \leq \# p \text { then } w \text { else tl } w \text { fi, } \\
& \text { if } k \leq \# p \text { then } r h s(p[k])++\operatorname{tl} v \text { else tl } v \text { fi, } s) \text { ) } \\
& \text { else } \operatorname{rec}^{\prime \prime}(\operatorname{next}(k, p, v), p, w, v, s) \mathrm{fi} \\
& \text { next: nat } x \text { sequ of prod } \times \text { sequ of symb } \rightarrow \text { nat } \\
& \operatorname{next}(k, p, v)=_{\mathrm{def}} \\
& \min \{i: \text { nat } I(k+1 \leq i \leq \# p \Delta \operatorname{lhs}(p[i])=\mathrm{hd} v) \nabla i=\# p+1\}
\end{aligned}
$$


where $r e c$ " can be further transformed into

$$
\begin{aligned}
& \operatorname{rec}^{\prime \prime}(k, p, w, v, s)=_{\text {def }} \\
& \text { if } s \vee k>\# p+1 \text { then } s \\
& \text { elsf } k \leq \# p \wedge v \neq[] \Delta \text { hd } v=\operatorname{lhs}(p[k]) \\
& \text { then } \operatorname{rec}^{\prime \prime}\left(\operatorname{next}(k, p, v), p, w, v, \operatorname{rec}^{\prime}(p, w, \operatorname{rhs}(p[k])++\mathrm{tl} v, s)\right) \\
& \text { elsf } k=\# p+1 \wedge w \neq[] \wedge v \neq[] \Delta \mathbf{h d} w=\mathbf{h d} v \\
& \text { then } \operatorname{rec}^{\prime \prime}\left(\operatorname{next}(k, p, v), p, w, v, \operatorname{rec}^{\prime}(p, \mathrm{tl} w, \mathrm{tl} v, s)\right) \\
& \text { else } \operatorname{rec}^{\prime \prime}(\operatorname{next}(k, p, v), p, w, v, s) \text { fi. }
\end{aligned}
$$

Similar to the Prolog interpreter, proving termination is slightly more difficult than in most of the other examples. In fact, as is known, absence of left-recursion in the grammar is necessary for the proof of termination.

Of course, we could have chosen an even "stronger" definition for next, viz.

$$
\begin{aligned}
& \operatorname{next}(k, p, v)=\operatorname{def} \\
& \min \{i: \operatorname{nat} \mid(k+1 \leq i \leq \# p \Delta \operatorname{lhs}(p[i])=\operatorname{hd} v \Delta \\
& \exists z: \operatorname{symb} \mid \operatorname{hd} v \vec{p} \operatorname{hd} w++z) \nabla i=\# p+1\}
\end{aligned}
$$

which obviously reflects the idea of LL(1) recognition.

\subsection{Variant 2: Non-deterministic choice}

\subsubsection{The rule}

$f(X)$ where

$f(x)==_{\text {def }}$

if $\{y: \mathrm{p} \mid \mathrm{Q}(x, y)\} \neq \varnothing$ then some $y: \mathrm{p} \mid \mathrm{Q}(x, y)$ else dummy fi

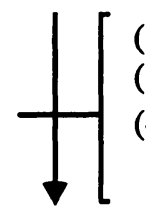

(1), (2), (5), (6), (7) as in general rule

(3) $\mathrm{P}(X, u, v) \Delta \mathrm{T}(u, v) \equiv$ true $+\mathrm{H}(u, v) \in\left\{y \mid \mathrm{Q}^{\prime}(u, v, y)\right\}=$ true

(4) $\mathrm{P}(X, u, v) \Delta \neg \mathrm{T}(u, v) \equiv$ true some $y: \mathbf{p} \mid \mathrm{Q}^{\prime}(u, v, y)=$ some $y: \mathbf{p} \mid \exists_{i=1 . . n(u, v)}(\mathrm{B}(i, u, v) \Delta$

$g(X)$ where $\left.\mathrm{Q}^{\prime}(\mathrm{K}(i, u, v), \mathrm{R}(i, u, v), y)\right)$

$g(x)==_{\text {def }} g^{\prime}(x$, E, dummy $)$

$g^{\prime}(u, v, s)=_{\text {def }}$ if $\mathrm{T}(u, v)$ then $\mathrm{H}(u, v)$ else $g^{\prime \prime}(\operatorname{next}(0, u, v), u, v, s)$ fi $g^{\prime \prime}(k, u, v, s)=_{\text {def }}$

if $s \neq$ dummy $\vee k>n(u, v)$ then $s$

elsf $\mathrm{B}(k, u, v)$ then $g$ " $\left(n \operatorname{ext}(k, u, v), u, v, g^{\prime}(\mathrm{K}(k, u, v), \mathrm{R}(k, u, v), s)\right)$ else $g^{\prime \prime}(n \operatorname{ext}(k, u, v), u, v, s)$ fi

\section{Syntactic constraints}

$\operatorname{KIND}[f, g]=\mathbf{m} \rightarrow \mathbf{n} \mid$ dummy 
$\operatorname{KIND}\left[g^{\prime}\right]=(u: \mathbf{m} \times v: \mathbf{r} \times s: \mathbf{n}|\mathbf{d u m m y}| \mathrm{P}(X, u, v)) \rightarrow \mathbf{n} \mid$ dummy

$\operatorname{KIND}\left[g^{\prime \prime}\right]=(k:$ nat $\times u: \mathbf{m} \times v: \mathbf{r} \times s: \mathbf{n} \mid$ dummy $\mid \mathrm{P}(X, u, v)) \rightarrow$ n I dummy

$\mathrm{KIND}[$ next $]=($ nat $\times \mathbf{m} \times \mathbf{r}) \rightarrow$ nat

\section{Proof (changes)}

a) Define

$a$ (C) $b==_{\operatorname{def}}$ if $\operatorname{def}(a)$ then $a$ [] $\operatorname{def}(b)$ then $b$ else dummy fi where

$\operatorname{def}($ some $y \mid \mathrm{Q})=\operatorname{def}\{y \mid \mathrm{Q}\} \neq \varnothing$

$\operatorname{def}(x)==_{\operatorname{def}} x \neq$ dummy.

(a2'): unfold $g^{\prime}$

(a3'): cond. (2); unfold (C)

b) (b2'): case-introduction

(b3'): $\supseteq$ for $=$

c) (c2'): $\supseteq$ for $\equiv$

(c3'): $v$-split in else-branch

(c5'): case introduction in else-branch; simplification using some $y \mid(B \vee C)$ $\equiv($ some $y \mid$ B) $\mathbb{C}$ (some $y \mid C)$

\subsubsection{An Application: Bottom-up parsing (of context-free grammars)}

Given a context-free grammar $G$ (as defined in the previous example) and a terminal word $w$, it is asked for computing a sequence of shift and reduce actions the application of which allows to reduce $w$ to the axiom $Z$ of $G$ provided such a sequence exists; if not, the result should be error.

For a formal specification of this problem we define nont, term, symb, and prod as in the previous example. The sequence of shift and reduce actions is defined by

srsequ $=_{\text {def }}$ " sequences of $\mathrm{S}(=$ shift $)$ and $\mathrm{R}(\mathrm{X}, \alpha)(=$ reduce with $(\mathrm{X}, \alpha))$ ».

The application of such a sequence to a terminal word is specified by

$$
\begin{aligned}
& \text { apply: sequ of prod } \times \text { sequ of symb } \times \text { sequ of term } \rightarrow \\
& \text { sequ of symb } \times \text { sequ of term } \\
& \text { apply }([], s t, w)==_{\operatorname{def}}(s t, w) \\
& \text { apply }(\mathrm{S}++s r s, s t, t++w)=_{\operatorname{def}} \text { apply }(s r s, s t++t, w) \\
& \text { apply }(\mathrm{R}(\mathrm{X}, \alpha)++s r s, s t++\alpha, w)={ }_{\operatorname{def}} \operatorname{apply}(s r s, s t++\mathrm{X}, w) .
\end{aligned}
$$

With these prerequisites, the original problem may be specified as follows:

parse $(P,[], W)$ where

parse: sequ of prod $\times$ sequ of symb $\times$ sequ of term $\rightarrow$ srsequ I error

$\operatorname{parse}(p, s t, w)=_{\mathrm{def}}$ 
if $\{y$ : srsequ $\mid \operatorname{apply}(y, s t, w)=([Z],[])\} \neq \varnothing$

then some $y$ : srsequ $\mid \operatorname{apply}(y, s t, w)=([Z],[])$ else error fi.

The result of rule application is

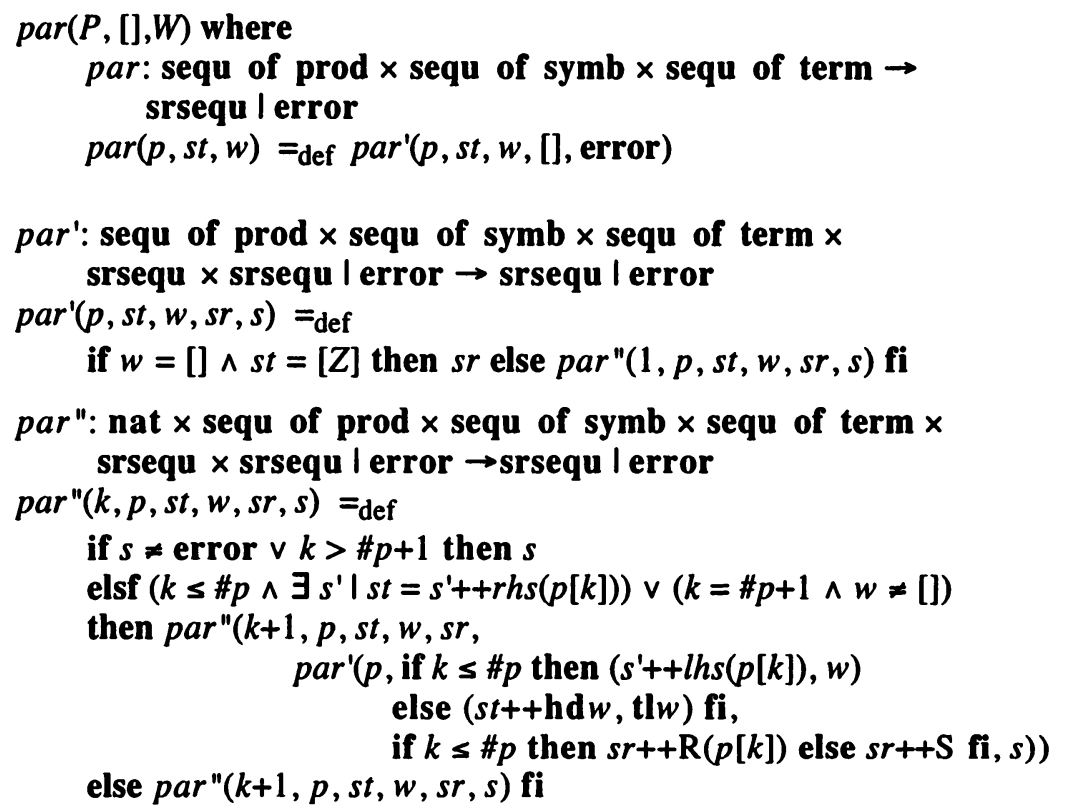

where par" can be further transformed into

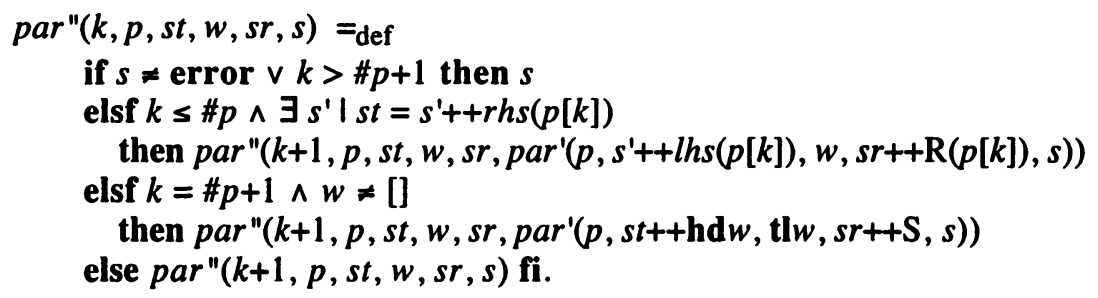

Again, as in the case of top-down recognition, additional constraints on the grammar are necessary for the proof of termination.

\subsection{Variant 3: A special instance of the rule}

\subsubsection{The rule}

Differently to the previous variants, we now consider a variant that is obtained by specializing the general rule by

- defining $n(u, v)=_{\text {def }} 1$ and $n \operatorname{ext}(k, u, v)==_{\text {def }} k+1$ 
- instantiating these definitions and simplifying the resulting expressions

- skipping the dependencies (on $i$ ) of the expressions $\mathrm{K}, \mathrm{R}$, and B.

This leads to the following specialization of our general rule:

$f(X)$ where

$f(x)==_{\operatorname{def}}\{r(X, y): \mathbf{n} \mid \mathrm{Q}(x, y)\}$

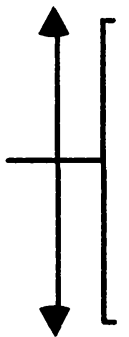

(0), (1), (2), (3) as in general rule

(4) $\mathrm{P}(X, u, v) \Delta \neg \mathrm{T}(u, v)=$ true $\left\{r(X, y) \mid \mathrm{Q}^{\prime}(u, v, y)\right\}=\left\{r(X, y) \mid \mathrm{B}(u, v) \Delta \mathrm{Q}^{\prime}(\mathrm{K}(u, v), \mathrm{R}(u, v), y)\right\}$

(6) $\mathrm{P}(X, u, v) \Delta \neg \mathrm{T}(u, v) \Delta \mathrm{B}(u, v) \equiv$ true r

$$
\mathrm{P}(X, \mathrm{~K}(u, v), \mathrm{R}(u, v))=\text { true }
$$

(7) $\mathrm{B}(u, v) \equiv$ true $+(\mathrm{R}(u, v)<v) \equiv$ true where WF-ORD $(\mathrm{r},<)$ or $(\mathrm{K}(u, v)<u) \equiv$ true where WF-ORD $(\mathrm{m},<)$

$g(X)$ where

$$
\begin{aligned}
& g(x)==_{\operatorname{def}} g^{\prime}(x, \mathrm{E}, \emptyset) \\
& g^{\prime}(u, v, s)==_{\operatorname{def}} \text { if } \mathrm{T}(u, v) \text { then } s \cup \mathrm{H}(u, v) \text { else } g^{\prime \prime}(1, u, v, s) \mathrm{fi} \\
& g^{\prime \prime}(k, u, v, s)==_{\operatorname{def}} \\
& \quad \text { if } k>1 \text { then } s \\
& \quad \text { elsf } \mathrm{B}(u, v) \text { then } g^{\prime \prime}\left(k+1, u, v, g^{\prime}(\mathrm{K}(u, v), \mathrm{R}(u, v), s)\right) \\
& \quad \text { else } g^{\prime \prime}(k+1, u, v, s) \text { fi }
\end{aligned}
$$

\section{Syntactic constraints}

as in general rule

Intuitively, under these particular constraints, one would expect a linear recursive output scheme. Indeed, this can be obtained by further transformation:

(a) $g$ " $(2, u, v, s)$

$$
\text { = [ instantiation ] }
$$

$s$

(b) $g^{\prime \prime}(1, u, v, s)$

= [ unfold $g$ " ]

if $1>1$ then $s$

elsf $\mathrm{B}(u, v)$ then $g^{\prime \prime}\left(2, u, v, g^{\prime}(\mathrm{K}(u, v), \mathrm{R}(u, v), s)\right)$ else $g^{\prime \prime}(2, u, v, s)$ fi

$\equiv$ [ simplification of conditional ]

if $\mathrm{B}(u, v)$ then $g^{\prime \prime}\left(2, u, v, g^{\prime}(\mathrm{K}(u, v), \mathrm{R}(u, v), s)\right)$ else $g^{\prime \prime}(2, u, v, s)$ fi

$=[(a)]$

if $\mathrm{B}(u, v)$ then $g^{\prime}(\mathrm{K}(u, v), \mathrm{R}(u, v), s)$ else $s$ fi 
(c) $g^{\prime}(u, v, s)$

$\equiv\left[\right.$ unfold $\left.g^{\prime}\right]$

if $\mathrm{T}(u, v)$ then $s \cup \mathrm{H}(u, v)$ else $g "(1, u, v, s)$ fi

$=[(\mathrm{b})]$

if $\mathrm{T}(u, v)$ then $s \cup \mathrm{H}(u, v)$

elsf $\mathrm{B}(u, v)$ then $g^{\prime}(\mathrm{K}(u, v), \mathrm{R}(u, v), s)$ else $s$ fi

(d) Define $g^{\prime \prime}(u, v)==_{\text {def }} g^{\prime}(u, v, \varnothing)$. Then

$g^{\prime \prime \prime}(u, v)$

$\equiv\left[\right.$ unfold $\left.g^{\prime \prime}\right]$

$g^{\prime}(u, v, \varnothing)$

= [ unfold $g^{\prime}$ as obtained in (b)]

if $\mathrm{T}(u, v)$ then $\emptyset \cup \mathrm{H}(u, v)$

elsf $\mathrm{B}(u, v)$ then $g^{\prime}(\mathrm{K}(u, v), \mathrm{R}(u, v), \emptyset)$ else $\emptyset \mathrm{fi}$

$=$ [ simplification; fold $g^{\prime \prime \prime}$ ]

if $\mathrm{T}(u, v)$ then $\mathrm{H}(u, v)$ elsf $\mathrm{B}(u, v)$ then $g^{\prime \prime}(\mathrm{K}(u, v), \mathrm{R}(u, v))$ else $\emptyset \mathrm{fi}$

Altogether, we have derived a new (linear recursive) output scheme:

$g(X)$ where

$g: \mathbf{m} \rightarrow$ set of $\mathbf{n}$

$g(x)=\operatorname{def} g^{\prime \prime \prime}(x, E)$ where

$g^{\prime \prime}:(u: \mathbf{m} \times v: \mathbf{r} \mid \mathrm{P}(X, u, v)) \rightarrow$ set of $\mathbf{n}$

$g^{\prime \prime \prime}(u, v)=\operatorname{def}$

if $\mathrm{T}(u, v)$ then $\mathrm{H}(u, v)$

elsf $\mathrm{B}(u, v)$ then $g^{\prime \prime}(\mathrm{K}(u, v), \mathrm{R}(u, v))$ else $\emptyset \mathrm{fi}$.

4.3.2 An application: Prime factors of a natural number $(\geq 2)$

Given a natural number $n(z 2)$, it is asked to compute the sequence of all prime factors of $n$ in increasing order.

The output type may be formally specified by

$$
\text { psequ }=_{\text {def }}(s: \text { natsequ } \mid \forall 1 \leq i \leq \# s \Delta \operatorname{prime}(s[i]))
$$

where,

prime: nat $\rightarrow$ bool

$\operatorname{prime}(x)={ }_{\text {def }} \| x$ is a prime number $»$.

Using, additionally,

prod: psequ $\rightarrow$ nat

$\operatorname{prod}(s p)={ }_{\operatorname{def}}$ if $s p=[]$ then 1 else hd $s p * \operatorname{prod}($ tlsp $)$ fi 
and

ordered: psequ $\rightarrow$ bool

$\operatorname{ordered}(s p)==_{\mathrm{def}} \| s p$ is increasingly ordered $»$

a complete formal specification of the problem is given by

all-primes $(N)$ where

all-primes: nat $\rightarrow$ set of psequ

all-primes $(n)==_{\text {def }}\{s p: \operatorname{psequ} \mid \operatorname{prod}(s p)=n \wedge \operatorname{ordered}(s p)\}$.

The result of rule application is (where div denotes integer division, and divides $(c, n)$ tests divisibility):

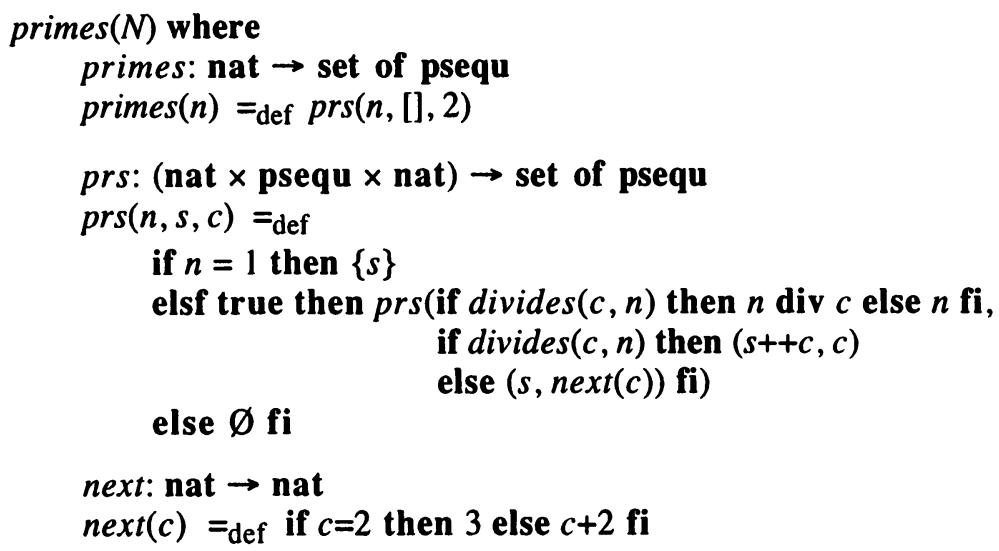

where prs can be further transformed (using simplification of conditional; distributivity of function call and conditional; simplification) into

$$
\begin{aligned}
& \operatorname{prs}(n, s, c)={ }_{\mathrm{def}} \\
& \quad \text { if } n=1 \text { then }\{s\} \\
& \quad \text { elsf } \operatorname{divides}(c, n) \text { then } \operatorname{prs}(n \operatorname{div} c, s++c, c) \text { else } \operatorname{prs}(n, s, n \operatorname{ext}(c)) \text { fi. }
\end{aligned}
$$

\subsection{Further variants}

Similar to variants 1 and 2 (where the initial specification asked for some expression over a set rather than the set itself), further variants could be given (and proved in an analogous way) to deal with other set expressions (e.g., min, max, or the like).

So far, we also have only considered variants where the alternatives resulting from the splitting of the computation according to applicability condition (4) are considered in an ordered way. Of course, we can also give analogous variants where the union

$$
\bigcup_{i=1 . n(u, v)}\left\{r(\mathrm{X}, y) \mid \mathrm{B}(i, u, v) \Delta \mathrm{Q}^{\prime}(K(i, u, v), \mathrm{R}(i, u, v), y)\right\}
$$


in condition (4) is replaced by

$$
\bigcup_{x \in m(u, v)}\left\{r(\mathrm{X}, y) \mid \mathrm{B}(x, u, v) \Delta \mathrm{Q}^{\prime}(\mathrm{K}(x, u, v), \mathrm{R}(x, u, v), y)\right\}
$$

and by using

$$
\begin{array}{ll}
m(u, v) \backslash\{x\} & \text { for } \operatorname{next}(k, u, v) \\
u \backslash\{x\} & \text { for } \mathrm{K}(x, u, v) \\
m(u, v)=\varnothing & \text { for } k>n(u, v) .
\end{array}
$$

Since, however, efficient computation requires an ordering of these alternatives anyhow, we have refrained from giving these variants explicitly.

\section{RELATED WORK AND CONCLUDING REMARKS}

As its main contribution, this paper has presented a powerful transformation rule to convert specifications of set-valued functions defined by set comprehension into executable functional implementations that basically show the behaviour of backtrack algorithms. From this central transformation rule we have furthermore derived several variants to cope with related problems and their solution by backtracking.

\subsection{Related work}

Early papers that dealt with the problem of backtracking from a methodical point of view are (Gerhart, Yelowitz, 1976) and (Broy;'Wirsing, 1980). Both papers give kind of functional program schemes to characterize and discuss the idea of backtracking, but do not investigate the problem of how to transform descriptive specifications into these functional schemes.

A treatment of backtracking as a solution to search problems which is fairly close to our approach can be found in the work of Doug Smith (cf., e.g., (Smith, 1986), (Smith, 1988), or (Smith, 1990) - also for further references). He gives various design strategies which achieve roughly the same effect as the application of our rule. Also the entirety of conditions required to apply these strategies is basically comparable to our applicability conditions. The main difference in the two approaches - apart from having a strategy there and a compact rule here - is in various technical aspects. Also, it is not quite clear how the variants we deal with fit into Smith's approach.

Of course, our rules presented in this paper also show many relationships to the rules presented in (Partsch, 1990). E.g., the rule " $\exists$-elimination by backtracking II" may be considered a specialized form of variant 2 presented here, and the rule "computing qualifiers for a finite set" is but a particular special case of our central rule. Moreover, a difference in methodically looking at the problem is worth mentioned here. Whereas in (Partsch, 1990) we primarily concentrate on solutions for the existence problem and present tactics to extend these solutions to also cover 
the "some" or the "set-of-all" problem, we started here the other way round which considerably reduces the amount of work for concrete applications.

\subsection{Concluding remarks}

In some sense, the problems our rule is able to deal with, can be viewed as generate-and-test problems to produce sets the elements of which are characterized by some predicate. Although the only real restriction for the application of the rule is provided by the applicability conditions, best use of the rule can be made for those problems where the elements of the solution are "complex" (i.e., not directly computable from the input) and where the search space is large (maybe infinite) and not easily enumerable. For "simpler" problems (not showing these characteristics) application of the rule is well possible, but usually will require additional effort to simplify the result.

It is obvious, that our rule is primarily aimed at languages with the initially mentioned semantic characteristics. For these, the rule offers a possibility to convert non-operational, descriptive specifications into operational ones - maybe as a first step towards further improving efficiency or switching to an imperative implementation. In the context of a non-strict, lazy functional language (such as Gofer), the value of our rule may be doubted, since in such a language our initial specifications may be directly executed. For all our examples, however, corresponding comparisons (with Gofer implementations of the respective program versions) have shown a substantial increase in efficiency (through application of our rule) also for a non-strict, lazy functional language.

The main problem in using the given transformation rules for concrete applications is in finding the appropriate instantiations required by the applicability conditions. Although some guidance (in finding instantiations) is given by the conditions themselves as well as by the remarks in Sect. 2, three major creative activities remain to be done:

- finding a suitable way of splitting the problem into subproblems (condition (4))

- inventing an appropriate invariant $P$ (conditions (2) and (5)), and

- defining a suitable ordering for the proof of termination (condition (6)).

Once a suitable instantiation has been found, the verification of the resulting proof obligations is straightforward and even gives potential for automation using a theorem prover. Experience (when dealing with all the examples given here and in (Achatz, Partsch, 1996)) has confirmed the assumption that the reverse of this statement also holds: Whenever complicated proof obligations resulted from some instantiation, it turned out that the real problem was with the instantiation rather than the proof obligation. 
Achatz, K. and Partsch, H.A. (1996) From Descriptive Specifications to Operational ones: A powerful transformation rule, its applications and variants. Faculty for Informatics, University of Ulm, Nr. 96-13, Ulmer InformatikBerichte, 1996.

Bauer, F.L. and Berghammer, R. and Broy, M. and Dosch, W. and Geiselbrechtinger, F. and Gnatz, R. and Hangel, E., Hesse, W.and KriegBrückner, B. and Laut, A. and Matzner, T. and Möller, B. and Nickl, F. and Partsch, H. and Pepper, P. and Samelson, K. and Wirsing, M. and Wössner, H. (1985) The Munich project CIP. Volume I: The wide spectrum language CIP-L. Lecture Notes in Computer Science 183, Springer, Berlin.

Bauer, F.L. and Möller and B., Partsch and H., Pepper, P. (1989) Programming by formal reasoning - computer-aided intuition-guided programming. IEEE Transactions on Software Engineering 15:2, 165-180.

Boiten, E.A. and Partsch, H.A. and Tuijnman, D. and Völker, N. (1992) How to produce correct software - an introduction to formal specification and program development by transformations. The Computer Journal 35:6, 547-554.

Broy, M. and Wirsing, M. (1980) Program development: from enumeration to backtracking. Information Processing letters 10:4,5, 193-197.

Gerhart, S.L. and Yelowitz, L. (1976) Control structure abstractions of the backtracking programming technique. Proc. 2nd Int. Conf. on Software Engineering, October 13-15, 1976, San Francisco, Ca., 44-49. Also: IEEE Transactions on Software Engineering 2:4, 285-292.

Partsch, H.A. (1990) Specification and transformation of programs - a formal approach to software development. Springer, Berlin

Smith, D.R. (1987) On the design of generate-and-test algorithms: subspace generators. Technical Report KES.U.86.6, Kestrel Institute, Palo Alto, Ca., 1986. Also: Meertens, L.G.L.T. (ed.): Program specification and transformation. Amsterdam: North-Holland, 207-220.

Smith, D.R. (1988) The structure and design of global search algorithms.Technical Report KES.U.87.12, Kestrel Institute, Palo Alto, Ca.Smith, D.R. (1990) KIDS: a semiautomatic program development system. IEEE Transactions on Software Engineering 16:9, 1024-1043.

\section{BIOGRAPHY}

Prof. Dr. Helmuth Partsch got his degree (Dipl.-Inform.) in informatics (with mathematics as a minor) from the Technical University of Munich. There, he also received his $\mathrm{Ph} D$ (Dr. rer. nat.) and his Habilitation degree. From 1986 till 1992 he held a chair for Software Engineering at the University of Nijmegen, the Netherlands. Since 1993 he heads the department Programming Methodology and Compiler Construction in the faculty for informatics at the University of Ulm, Germany. Prof. Partsch's research interests are in formal software development, 
notably in formal specification and program constuction by transformations. A particular focus of his research activities is the integration of formal methods with traditional Software Engineering techniques.

Klaus Achatz got his degree (Dipl.-Math.) in mathematics (with informatics as a minor) from the University of Augsburg. Since 1993, he is a research and teaching assistant in the department Programming Methodology and Compiler Construction in the faculty for informatics at the University of Ulm. Klaus Achatz's research interests are in the integration of formal concepts with (object-oriented) Software Engineering methods. 